\title{
Homoclinic Orbits for a Class of Subquadratic Second Order Hamiltonian Systems
}

\author{
Li-Li Wan \\ School of Science, Southwest University of Science and Technology, Mianyang, Sichuan 621010, China \\ Correspondence should be addressed to Li-Li Wan; 15882872311@163.com
}

Received 13 November 2013; Accepted 19 January 2014; Published 4 March 2014

Academic Editors: G. L. Karakostas and C. Wang

Copyright (C) $2014 \mathrm{Li}-\mathrm{Li}$ Wan. This is an open access article distributed under the Creative Commons Attribution License, which permits unrestricted use, distribution, and reproduction in any medium, provided the original work is properly cited.

The existence and multiplicity of homoclinic orbits are considered for a class of subquadratic second order Hamiltonian systems $\ddot{q}(t)-L(t) q(t)+\nabla W(t, q(t))=0$. Recent results from the literature are generalized and significantly improved. Examples are also given in this paper to illustrate our main results.

\section{Introduction and Main Results}

In this paper, we will study the existence and multiplicity of homoclinic orbits for the second order Hamiltonian systems of the type:

$$
\ddot{q}(t)-L(t) q(t)+\nabla W(t, q(t))=0,
$$

where $L \in C\left(\mathbb{R}, \mathbb{R}^{\mathbb{N}^{2}}\right)$ is a symmetric matrix valued function and $W \in C^{1}\left(\mathbb{R} \times \mathbb{R}^{\mathbb{N}}, \mathbb{R}\right)$. As usual, we say that $q$ is a nontrivial homoclinic orbit (to 0 ) if $q \in C^{2}\left(\mathbb{R}, \mathbb{R}^{\mathbb{N}}\right), q \neq 0$ and $q(t) \rightarrow 0$ as $|t| \rightarrow \infty$ (see [1]). In the following, $(\cdot, \cdot): \mathbb{R}^{\mathbb{N}} \times \mathbb{R}^{\mathbb{N}} \mapsto \mathbb{R}$ denotes the standard inner product in $\mathbb{R}^{\mathbb{N}}$ and $|\cdot|$ is the induced norm.

Hamiltonian system theory, a classical as well as a modern study area, widely consists in mathematical sciences, life sciences, and various aspects of social science. Lots of mechanical and field theory models even exist in the form of Hamiltonian system. Solutions of Hamiltonian system can be divided into periodic solution, subharmonic solution, homoclinic orbit, heteroclinic orbit, and so on. Homoclinic orbit was discovered among the models of nonlinear dynamical system and affects the nature of the whole system significantly. Starting from the Poincaré era, the study of homoclinic orbits in the nonlinear dynamical system exploits Perturbation method mainly. It is not until the recent decade that variational principle has been widely used. The existence and multiplicity of homoclinic orbits for the second order Hamiltonian systems have been extensively investigated in many recent papers (see [1-16]). The main feature of the problem is the lack of global compactness due to unboundedness of domain. To overcome the difficulty, many authors have considered the periodic case, autonomous case, or asymptotically periodic case (see [1-4]). Some papers treat the coercive case (see [5-8]). Recently, the symmetric case has been dealt with (see [9-11]). Compared with the superquadratic case, the case that $W(t, x)$ is subquadratic as $|x| \rightarrow+\infty$ has been considered only by a few authors. As far as the author is aware, the author in [5] first discussed the subquadratic case. Later, the authors in [12] dealt with this case by use of a standard minimizing argument, which is the following theorem.

Theorem 1 (see [12]). Assume that $L$ and $W$ satisfy the following conditions:

$\left(A_{1}\right) L \in C\left(\mathbb{R}, \mathbb{R}^{\mathbb{N}^{2}}\right)$ is a symmetric and positive definite matrix for all $t \in \mathbb{R}$ and there is a continuous function $\alpha: \mathbb{R} \rightarrow \mathbb{R}^{+}$such that $(L(t) x, x) \geq \alpha(t)|x|^{2}$ and $\alpha(t) \rightarrow+\infty$ as $|t| \rightarrow+\infty$;

$\left(A_{2}\right) W(t, x)=a(t)|x|^{\gamma}$, where $a: \mathbb{R} \rightarrow \mathbb{R}^{+}$is a continuous function such that $a \in L^{2}(\mathbb{R}, \mathbb{R}) \bigcap L^{2 /(2-\gamma)}(\mathbb{R}, \mathbb{R})$ and $1<\gamma<2$ is a constant.

Then, (1) has at least one nontrivial homoclinic orbit.

Subsequently, the condition $\left(A_{2}\right)$ was generalized, respectively, in $[13,14]$ by the following conditions: 
$\left(A_{3}\right) W(t, x)=a(t)|x|^{\gamma}$, where $a: \mathbb{R} \rightarrow \mathbb{R}^{+}$is a continuous function such that $a \in L^{2 /(2-\gamma)}(\mathbb{R}, \mathbb{R})$ and $1<\gamma<2$ is a constant.

$\left(A_{4}\right) a(t)|x|^{\gamma} \leq(\nabla W(t, x), x), \mid\left(\left.\nabla W(t, x)|\leq b(t)| x\right|^{\gamma-1}+\right.$ $c(t)|x|^{\delta-1}$, where $a, b, c: \mathbb{R} \rightarrow \mathbb{R}^{+}$are continuous functions such that $a, b \in L^{2 /(2-\gamma)}(\mathbb{R}, \mathbb{R}), c \in$ $L^{2 /(2-\delta)}(\mathbb{R}, \mathbb{R})$, and $1<\gamma<2,1<\delta<2$ are constants, $W(t, 0)=0, W(t, x)=W(t,-x)$ for all $(t, x) \in\left(\mathbb{R}, \mathbb{R}^{\mathbb{N}}\right)$

The authors in $[13,14]$ got infinitely many homoclinic orbits by using the variant fountain theorem in [17].

Motivated by these papers, we also consider the subquadratic case. Main results are the following theorems.

Theorem 2. Assume that $\left(A_{1}\right)$ is satisfied and the following conditions hold:

$\left(W_{1}\right)$ there exists a constant $1 \leq \gamma<2$ such that $|W(t, x)| \leq$ $a(t) b(|x|)|x|^{\gamma}$ for all $t \in \mathbb{R}$ and $x \in \mathbb{R}^{\mathbb{N}}$, where $a \in$ $C\left(\mathbb{R}, \mathbb{R}^{+}\right) \bigcap L^{2 /(2-\gamma)}(\mathbb{R}, \mathbb{R})$ and $b \in C\left(\mathbb{R}^{+}, \mathbb{R}^{+}\right) ;$

$\left(W_{2}\right)|\nabla W(t, x)| \leq a(t) d(|x|)|x|^{\gamma-1}$ for all $\in \mathbb{R}$ and $x \in \mathbb{R}^{\mathbb{N}}$, where $d \in C\left(\mathbb{R}^{+}, \mathbb{R}^{+}\right)$;

$\left(W_{3}\right) \lim _{|x| \rightarrow+\infty} W(t, x) /|x|^{2}=0$ uniformly for $t \in \mathbb{R}$;

$\left(W_{4}\right)$ there exists a constant $\delta_{0}>0$ such that $W(t, x) \geq$ $c_{0} a(t)|x|^{\gamma}$ for all $t \in \mathbb{R}$ and $|x| \leq \delta_{0}$, where $c_{0}$ is a positive constant.

Then, (1) has at least one nontrivial homoclinic orbit.

Remark 3. Theorem 2 generalizes Theorem 1 (see [12]). Obviously, the condition $\left(A_{2}\right)$ is stronger than the conditions $\left(W_{1}\right)-\left(W_{4}\right)$. On the other hand, there are functions $W$ satisfying our Theorem 2 and not satisfying the corresponding result in [12]. For example, let

$$
W_{0}=a(t)|x|^{\gamma}\left[\ln \left(1+|x|^{2}\right)+1\right], \quad \forall t \in \mathbb{R}, x \in \mathbb{R}^{\mathbb{N}},
$$

where $a \in C\left(\mathbb{R}, \mathbb{R}^{+}\right) \bigcap L^{2 /(2-\gamma)}(\mathbb{R}, \mathbb{R})$ and $1 \leq \gamma<2$. Moreover, our result is different from the corresponding results in $[5,15]$, since $W_{0}$ does not satisfy conditions $\left(W_{3}\right)$ and $\left(W_{7}\right)$ in [5] and our condition $\left(A_{1}\right)$ on $L$ is different from the condition $\left(L^{\prime}\right)$ in [15].

Theorem 4. Assume that $\left(A_{1}\right),\left(W_{1}\right)-\left(W_{4}\right)$ are satisfied and the following condition holds:

$$
\begin{gathered}
\left(W_{5}\right) W(t, x) \geq 0 \text { and } W(t, x)=W(t,-x) \text { for all } t \in \mathbb{R} \text { and } \\
x \in \mathbb{R}^{\mathbb{N}} .
\end{gathered}
$$

Then, (1) has infinitely many homoclinic orbits.

Remark 5. Theorem 4 generalizes Theorem 1.2 in [13]. It is easy to see that the condition $\left(A_{3}\right)$ is stronger than our conditions $\left(W_{1}\right)-\left(W_{5}\right)$. On the other hand, there are functions $W$ satisfying Theorem 4 and not satisfying the corresponding result in [13]. For example, let

$$
W_{1}=a(t)|x|^{\gamma}\left[\ln \left(1+|x|^{2}\right)+1\right], \quad \forall t \in \mathbb{R}, x \in \mathbb{R}^{\mathbb{N}},
$$

where $a \in C\left(\mathbb{R}, \mathbb{R}^{+}\right) \bigcap L^{2 /(2-\gamma)}(\mathbb{R}, \mathbb{R})$ and $1 \leq \gamma<2$.
Theorem 6. Assume that $\left(A_{1}\right),\left(W_{3}\right)-\left(W_{5}\right)$ are satisfied and the following conditions hold:

$\left(W_{6}\right)$ there exist constants $1 \leq \gamma_{k}<2$ and $m \in \mathbb{N}$ such that $|W(t, x)| \leq \sum_{k=1}^{m} a_{k}(t) b_{k}(|x|)|x|^{\gamma_{k}}$ for all $t \in \mathbb{R}$ and $x \in$ $\mathbb{R}^{\mathbb{N}}$, where $a_{k} \in C\left(\mathbb{R}, \mathbb{R}^{+}\right) \bigcap L^{2 /\left(2-\gamma_{k}\right)}(\mathbb{R}, \mathbb{R})$ and $b_{k} \in$ $C\left(\mathbb{R}^{+}, \mathbb{R}^{+}\right)$;

$\left(W_{7}\right)|\nabla W(t, x)| \leq \sum_{k=1}^{m} a_{k}(t) d_{k}(|x|)|x|^{\gamma_{k}-1}$ for all $t \in \mathbb{R}$ and $x \in \mathbb{R}^{\mathbb{N}}$, where $d_{k} \in C\left(\mathbb{R}^{+}, \mathbb{R}^{+}\right)$.

Then, (1) has infinitely many homoclinic orbits.

Remark 7. Theorem 6 generalizes Theorem 1.2 in [14]. It is obvious that the condition $\left(A_{4}\right)$ is stronger than our conditions $\left(W_{3}\right)-\left(W_{7}\right)$. Besides, there are functions $W$ satisfying our Theorem 6 and not satisfying the corresponding result in [14]. For example, let

$$
\begin{array}{r}
W_{2}=\left(a_{1}(t)|x|^{\gamma_{1}}+a_{2}(t)|x|^{\gamma_{2}}\right)\left[\ln \left(1+|x|^{2}\right)+1\right], \\
\forall t \in \mathbb{R}, x \in \mathbb{R}^{\mathbb{N}},
\end{array}
$$

where $a_{k} \in C\left(\mathbb{R}, \mathbb{R}^{+}\right) \bigcap L^{2 /\left(2-\gamma_{k}\right)}(\mathbb{R}, \mathbb{R}), 1 \leq \gamma_{k}<2$ and $k=$ 1,2 .

Remark 8. We should point out that there exist errors in the proofs of $[13,14]$. On one hand, in Lemma 2.2, in [13], $\left\|u_{k}-u\right\|_{2} \rightarrow 0$ can not imply that $\sum_{k=1}^{\infty}\left\|u_{k}-u\right\|_{2}<$ $+\infty$ since the convergence of the general term is only the necessary but not the sufficient condition for the convergence of the progression. On the other hand, the proof of Lemma 2.2 in [14] is not like the proof of Lemma 2.2 in [12] as the authors said. It is easy to see that $a \in L^{2}(\mathbb{R}, \mathbb{R})$ plays an important role in the proof of Lemma 2.2 in [12], however, $b, c$ in condition $\left(A_{4}\right)$ do not belong to $L^{2}(\mathbb{R}, \mathbb{R})$. In the proofs of our results, we take some methods to avoid such mistakes.

\section{Proof of Main Results}

Let

$$
E=\left\{q \in H^{1}\left(\mathbb{R}, \mathbb{R}^{\mathbb{N}}\right) \mid \int_{\mathbb{R}}\left(|\dot{q}|^{2}+(L(t) q, q)\right) d t<+\infty\right\} .
$$

Then, $E$ is a Hilbert space with the norm given by

$$
\|q\|=\left(\int_{\mathbb{R}}\left(|\dot{q}|^{2}+(L(t) q, q)\right) d t\right)^{1 / 2} .
$$

Obviously, $E$ is continuously embedded in $L^{p}\left(\mathbb{R}, \mathbb{R}^{\mathbb{N}}\right)$ for $p \in$ $[2, \infty]$. Thus, we have

$$
\|q\|_{L^{p}} \leq \eta_{p}\|q\| \text { for } p \in[2, \infty],
$$

where $\eta_{p}>0$. For $q \in E$, let

$$
\begin{aligned}
I(q)= & \frac{1}{2} \int_{\mathbb{R}}\left(|\dot{q}|^{2}+(L(t) q, q)\right) d t \\
& -\int_{\mathbb{R}} W(t, q) d t=\frac{1}{2}\|q\|^{2}-\int_{\mathbb{R}} W(t, q) d t .
\end{aligned}
$$


In the following, we always denote by $c_{i}(i \in \mathbb{N})$ any suitable positive constant.

Lemma 9 (see [5]). Assume that $L$ satisfies $\left(A_{1}\right)$. Then, $E$ is compactly embedded in $L^{p}\left(\mathbb{R}, \mathbb{R}^{\mathbb{N}}\right)$ for $p \in[2, \infty]$.

Lemma 10. Under the conditions of $\left(W_{1}\right)-\left(W_{3}\right)$, one has that $I \in C^{1}(E, \mathbb{R})$ and that

$$
\left\langle I^{\prime}(q), v\right\rangle=\int_{\mathbb{R}}((\dot{q}, \dot{v})+(L(t) q, v)-(\nabla W(t, q), v)) d t
$$

$\forall q, v \in E$.

Proof. Let $J(q)=\int_{\mathbb{R}} W(t, q) d t$ for all $q \in E$. For any $\varepsilon>0$, it follows from $\left(W_{1}\right)$ and $\left(W_{3}\right)$ that

$$
|W(t, x)| \leq \varepsilon|x|^{2}+c_{1} a(t)|x|^{\gamma}, \quad \forall t \in \mathbb{R}, x \in \mathbb{R}^{\mathbb{N}} .
$$

Then, by the Hölder inequality and (7), we have

$$
\begin{aligned}
\left|\int_{\mathbb{R}} W(t, q) d t\right| & \leq \varepsilon \eta_{2}^{2}\|q\|^{2}+c_{1}\|a\|_{L^{2 /(2-\gamma)}}\|q\|_{L^{2}}^{\gamma} \\
& \leq \varepsilon \eta_{2}^{2}\|q\|^{2}+c_{2}\|a\|_{L^{2 /(2-\gamma)}}\|q\|^{\gamma},
\end{aligned}
$$

for all $q \in E$. Therefore, $J$ is well defined. Moreover, $J \in$ $C^{1}(E, \mathbb{R})$ and

$$
\left\langle J^{\prime}(q), v\right\rangle=\int_{\mathbb{R}}(\nabla W(t, q), v) d t, \quad \forall q, v \in E .
$$

In fact, for any given $q \in E$, by $\left(W_{2}\right)$, the Hölder inequality, and (7), one has

$$
\begin{aligned}
\left|\int_{\mathbb{R}}(\nabla W(t, q), v) d t\right| & \leq c_{3} \int_{\mathbb{R}} a(t)|q|^{\gamma-1}|v| d t \\
& \leq c_{3}\left(\int_{\mathbb{R}} a^{2}(t)|q|^{2 \gamma-2} d t\right)^{1 / 2}\|v\|_{L^{2}} \\
& \leq c_{3}\|a\|_{L^{2 /(2-\gamma)}}\|q\|_{L^{2}}^{\gamma-1}\|v\|_{L^{2}} \\
& \leq c_{4}\|a\|_{L^{2 /(2-\gamma)}}\|v\|,
\end{aligned}
$$

for all $v \in E$. For any $q, v \in E$, by the mean value theorem, we have

$$
\begin{gathered}
\int_{\mathbb{R}} W(t, q+v) d t-\int_{\mathbb{R}} W(t, q) d t \\
=\int_{\mathbb{R}}(\nabla W(t, q+h v), v) d t,
\end{gathered}
$$

where $h(t) \in(0,1)$. Besides, for any given $q \in E$, by $\left(W_{2}\right)$, the Hölder inequality, and (7), there exists a positive constant $\theta_{0}$ such that

$$
\begin{aligned}
& \left|\int_{\mathbb{R}}(\nabla W(t, q+h v), v) d t\right| \\
& \quad \leq c_{5} \int_{\mathbb{R}} a(t)|q+h v|^{\gamma-1}|v| d t \\
& \quad \leq c_{5}\left(\int_{\mathbb{R}} a^{2}(t)|q+h v|^{2 \gamma-2} d t\right)^{1 / 2}\|v\|_{L^{2}} \\
& \quad \leq c_{5}\|a\|_{L^{2 /(2-\gamma)}}\|q+h v\|_{L^{2}}^{\gamma-1}\|v\|_{L^{2}} \\
& \quad \leq c_{6}\|a\|_{L^{2 /(2-\gamma)}}\|v\|,
\end{aligned}
$$

for all $\|v\| \leq \theta_{0}$. The combination of (13)-(15) shows

$$
\begin{aligned}
& \int_{\mathbb{R}} W(t, q+v) d t \\
& \quad-\int_{\mathbb{R}} W(t, q) d t-\int_{\mathbb{R}}(\nabla W(t, q), v) d t \longrightarrow 0,
\end{aligned}
$$

as $v \rightarrow 0$ in $E$, which gives (12) and (9) immediately. In addition, $J$ is weakly continuous. In fact, let $q_{n} \rightarrow q$ in $E$. By Lemma $9, q_{n} \rightarrow q$ in $L^{2}\left(\mathbb{R}, \mathbb{R}^{\mathbb{N}}\right)$ and $L^{\infty}\left(\mathbb{R}, \mathbb{R}^{\mathbb{N}}\right)$. Since $|q(t)| \rightarrow 0$ as $|t| \rightarrow \infty$, there exists a positive constant $R_{1}$ such that

$$
\left|q_{n}\right| \leq 1, \quad|q| \leq 1, \quad \forall|t| \geq R_{1} .
$$

It follows from $\left(W_{1}\right)$ and the Hölder inequality such that

$$
\begin{aligned}
& \int_{|t| \geq R_{1}}\left|W\left(t, q_{n}\right)-W(t, q)\right| d t \\
& \quad \leq c_{7} \int_{|t| \geq R_{1}} a(t)\left(\left|q_{n}\right|^{\gamma}+|q|^{\gamma}\right) d t \\
& \quad \leq c_{7}\|a\|_{L^{2 /(2-\gamma)}}\left[\left(\int_{|t| \geq R_{1}}\left|q_{n}\right|^{2} d t\right)^{\gamma / 2}+\left(\int_{|t| \geq R_{1}}|q|^{2} d t\right)^{\gamma / 2}\right] .
\end{aligned}
$$

Therefore, for any $\varepsilon>0$, since $q_{n} \rightarrow q$ in $L^{2}\left(\mathbb{R}, \mathbb{R}^{\mathbb{N}}\right)$, one can take $R_{2} \geq R_{1}$ such that

$$
\int_{|t| \geq R_{2}}\left|W\left(t, q_{n}\right)-W(t, q)\right| d t<\frac{\varepsilon}{2}, \quad \forall n \in \mathbb{N} .
$$

On the other hand, one has

$$
\int_{|t|<R_{2}} W\left(t, q_{n}\right) d t \longrightarrow \int_{|t|<R_{2}} W(t, q) d t \quad \text { as } n \longrightarrow+\infty \text {. }
$$

Hence, there exists $n_{0} \in \mathbb{N}$ such that

$$
\left|J\left(q_{n}\right)-J(q)\right|<\varepsilon
$$

proving the weak continuity of $J$. Moreover, $J^{\prime}$ is compact for $J$ is weakly continuous (see [5]). 
Lemma 11. Under the conditions of $\left(W_{3}\right),\left(W_{6}\right)$, and $\left(W_{7}\right)$, one has that $I \in C^{1}(E, \mathbb{R})$ and that

$$
\left\langle I^{\prime}(q), v\right\rangle=\int_{\mathbb{R}}((\dot{q}, \dot{v})+(L(t) q, v)-(\nabla W(t, q), v)) d t,
$$$$
\forall q, v \in E .
$$

Proof. Since the proof is exactly similar to the proof of Lemma 10, we omit it here.

By Lemmas 10 and 11, it is routine to verify that any critical point of $I$ on $E$ is a classical solution of (1) with $q( \pm \infty)=0$. Now, we state the critical point theorem used in [12].

Lemma 12 (see [18]). Let $E$ be a real Banach space and let $u s$ have $I \in C^{1}(E, \mathbb{R})$ satisfying the $(P S)$ condition. If $I$ is bounded from below, then

$$
c \equiv \inf _{E} I
$$

is a critical value of $I$.

Proof of Theorem 2. We divide our proof into three steps.

Step 1. I is bounded from below. In fact, using (11), we have

$$
\begin{aligned}
I(q) & =\frac{1}{2}\|q\|^{2}-\int_{\mathbb{R}} W(t, q) d t \\
& \geq \frac{1}{2}\|q\|^{2}-\varepsilon \eta_{2}^{2}\|q\|^{2}-c_{2}\|a\|_{L^{2 /(2-\gamma)}}\|q\|^{\gamma},
\end{aligned}
$$

for all $q \in E$. Since $1 \leq \gamma<2$ and $\varepsilon$ is an arbitrary positive constant, $I(q) \rightarrow+\infty$ as $\|q\| \rightarrow+\infty$ and then $I$ is bounded from below.

Step 2. I satisfies the (PS) condition. Assume that $\left\{q_{k}\right\}_{k \in \mathbb{N}} \subset E$ is a sequence such that

$$
I\left(q_{k}\right) \text { is bounded and } I^{\prime}\left(q_{k}\right) \longrightarrow \text { as } k \longrightarrow+\infty \text {. }
$$

By (11), one gets

$$
\begin{aligned}
& \frac{1}{2}\left\|q_{k}\right\|^{2}-I\left(q_{k}\right) \\
& \quad=\int_{\mathbb{R}} W\left(t, q_{k}\right) d t \leq \varepsilon \eta_{2}^{2}\left\|q_{k}\right\|^{2}+c_{2}\|a\|_{L^{2 /(2-\gamma)}}\left\|q_{k}\right\|^{\gamma},
\end{aligned}
$$

for all $k \in \mathbb{N}$. Since $1 \leq \gamma<2$ and $\varepsilon$ is an arbitrary positive constant, it is obvious that $\left\{q_{k}\right\}$ is bounded. Noting that $J^{\prime}$ is compact, a routine verification shows that $\left\{q_{k}\right\}$ has a convergent subsequence, proving the (PS) condition. Now, by Lemma 12 , there is a $q_{0} \in E$ such that

$$
I\left(q_{0}\right)=\inf _{E} I(q), \quad I^{\prime}\left(q_{0}\right)=0 .
$$

Step 3. $q_{0}$ is nontrivial. In fact, let

$$
e(t)= \begin{cases}|\sin t| e_{1}, & t \in[-\pi, \pi], \\ 0, & t \in \mathbb{R} \backslash[-\pi, \pi],\end{cases}
$$

where $e_{1}=(1,0, \ldots, 0)$. By $\left(W_{4}\right)$ one obtains

$$
\begin{aligned}
I(\xi e) & =\frac{\xi^{2}}{2}\|e\|^{2}-\int_{-\pi}^{\pi} W(t, \xi e) d t \\
& \leq \frac{\xi^{2}}{2}\|e\|^{2}-c_{0} \xi^{\gamma} \int_{-\pi}^{\pi} a(t)|e|^{\gamma} d t,
\end{aligned}
$$

for $|\xi| \leq \delta_{0}$. Since $1 \leq \gamma<2$, we can choose $\xi$ small enough such that $I(\xi e)<0$. Hence, $q_{0} \not \equiv 0$. The proof is complete.

In order to prove Theorems 4 and 6, we need the Dual fountain theorem. For the reader's convenience, we recall it here.

Lemma 13 (see [19]). Let $E$ be a Banach space with the norm $\|\cdot\|$ and $E=\overline{\bigoplus_{j \in N} X_{j}}$ with $\operatorname{dim} X_{j}<+\infty$. For any $k \in \mathbb{N}$, define

$$
Y_{k}=\bigoplus_{j=1}^{j=k} X_{j}, \quad Z_{k}=\overline{\bigoplus_{j=k}^{\infty} X_{j}}
$$

Assume that

$\left(B_{1}\right)$ the compact group $G$ acts isometrically on $E$. The spaces $X_{j}$ are invariant and there exists a finite dimensional space $V$ such that, for every $j \in \mathbb{N}, X_{j} \simeq V$ and the action of $G$ on $V$ is admissible.

Besides, let $I \in \mathscr{C}^{1}(E, \mathbb{R})$ be an invariant functional. If for every $k \geq k_{0}$, there exist $\rho_{k}>r_{k}>0$ such that

$$
\begin{aligned}
& \left(B_{2}\right) a_{k}=\inf _{q \in Z_{k},\|q\|=\rho_{k}} I(q) \geq 0 ; \\
& \left(B_{3}\right) b_{k}=\max _{q \in Y_{k},\|q\|=r_{k}} I(q)<0 ; \\
& \left(B_{4}\right) d_{k}=\inf _{q \in Z_{k},\|q\| \leq \rho_{k}} I(q) \rightarrow 0 \text { as } k \rightarrow+\infty ; \\
& \left(B_{5}\right) \text { I satisfies the }(P S)_{c}^{*} \text { condition for every } c \in\left[d_{k_{0}}, 0[.\right.
\end{aligned}
$$

Then, I has a sequence of negative critical values converging to 0 .

Proof of Theorem 4. Let $\left\{e_{j}\right\}_{j \in \mathbb{N}}$ be the completely orthogonal basis of $E$ and $X_{j}=\mathbb{R} e_{j}$. Then, $\operatorname{dim} X_{j}<+\infty$ and $E=$ $\overline{\bigoplus_{j \in N} X_{j}}$. For any $k \in \mathbb{N}$, define

$$
Y_{k}=\bigoplus_{j=1}^{j=k} X_{j}, \quad Z_{k}=\overline{\bigoplus_{j=k}^{\infty} X_{j}}
$$

In the following, we will check that all conditions in Lemma 13 are satisfied and we divide our proof into several steps.

Step 1. Conditions $\left(B_{2}\right)$ and $\left(B_{4}\right)$ hold. Let

$$
\mu_{k}=\sup _{q \in Z_{k},\|q\|=1}\|q\|_{L^{2}}
$$

We claim that $\mu_{k} \rightarrow 0$ as $k \rightarrow+\infty$. In fact, it is obvious that $0<\mu_{k+1} \leq \mu_{k}$ and then $\mu_{k} \rightarrow \mu_{0}$ for some $\mu_{0} \geq 0$ 
as $k \rightarrow+\infty$. For every $k \in \mathbb{N}$, there is $q_{k} \in Z_{k}$ such that $\left\|q_{k}\right\|=1$ and $\left\|q_{k}\right\|_{L^{2}}>\mu_{k} / 2$. It follows from the definition of $Z_{k}$ that $q_{k} \rightarrow 0$ in $E$. Then, by Lemma 9, we have $q_{k} \rightarrow 0$ in $L^{2}\left(\mathbb{R}, \mathbb{R}^{\mathbb{N}}\right)$ as $k \rightarrow+\infty$ and thus $\mu_{0}=0$. Take $\varepsilon=1 / 4 \eta_{2}^{2}$ in (11), then for all $q \in Z_{k}$, one gets

$$
\begin{aligned}
I(q) & =\frac{1}{2}\|q\|^{2}-\int_{\mathbb{R}} W(t, q) d t \\
& \geq \frac{1}{2}\|q\|^{2}-\varepsilon \eta_{2}^{2}\|q\|^{2}-c_{1}\|a\|_{L^{2 /(2-\gamma)}}\|q\|_{L^{2}}^{\gamma} \\
& =\frac{1}{2}\|q\|^{2}-\frac{1}{4}\|q\|^{2}-c_{1}\|a\|_{L^{2 /(2-\gamma)}}\|q\|_{L^{2}}^{\gamma} \\
& \geq \frac{1}{4}\|q\|^{2}-c_{1} \mu_{k}^{\gamma}\|a\|_{L^{2 /(2-\gamma)}}\|q\|^{\gamma} .
\end{aligned}
$$

Since $1 \leq \gamma<2$, there exists $\rho_{k}>0$ such that

$$
\begin{gathered}
a_{k}=\inf _{q \in Z_{k},\|q\|=\rho_{k}} I(q) \geq 0, \\
d_{k}=\inf _{q \in Z_{k},\|q\| \leq \rho_{k}} I(q) \geq-c_{1} \mu_{k}^{\gamma}\|a\|_{L^{2 /(2-\gamma)}\|q\|^{\gamma} .}
\end{gathered}
$$

Similar to the third step in the proof of Theorem 2, we can show that $d_{k} \leq 0$. Since $\mu_{k} \rightarrow 0$ as $k \rightarrow+\infty$, we get $d_{k} \rightarrow 0$ as $k \rightarrow+\infty$.

Step 2. Condition $\left(B_{3}\right)$ holds. We claim that there exists $\varepsilon_{1}>0$ such that

$$
\text { meas }\left\{t \in \mathbb{R}: a(t)|q(t)|^{\gamma} \geq \varepsilon_{1}\|q\|^{\gamma}\right\} \geq \varepsilon_{1}, \quad \forall q \in Y_{k} \backslash\{0\} .
$$

If not, there exists a sequence $\left\{q_{m}\right\}_{m \in \mathbb{N}} \subset Y_{k}$ with $\left\|q_{m}\right\|=1$ such that

$$
\text { meas }\left\{t \in \mathbb{R}: a(t)\left|q_{m}(t)\right|^{\gamma} \geq \frac{1}{m}\right\}<\frac{1}{m},
$$

for all $m \in \mathbb{N}$. Since we have $\operatorname{dim} Y_{k}<+\infty$, it follows from the compactness of the unit sphere of $Y_{k}$ that, going to a subsequence if necessary, $q_{m}$ converges to some $q^{*}$ in $Y_{k}$ and $\left\|q^{*}\right\|=1$. By the equivalence of the norms on the finitedimensional space $Y_{k}$, one has

$$
\int_{\mathbb{R}} a(t)\left|q_{m}-q^{*}\right|^{\gamma} d t \leq\|a\|_{L^{2 /(2-\gamma)}}\left(\int_{\mathbb{R}}\left|q_{m}-q^{*}\right|^{2} d t\right)^{\gamma / 2} \longrightarrow 0,
$$

as $m \rightarrow+\infty$. It is easy to check that there exist $\delta_{1}>0$ and $\delta_{2}>0$ such that

$$
\text { meas }\left\{t \in \mathbb{R}: a(t)\left|q^{*}(t)\right|^{\gamma} \geq \delta_{1}\right\} \geq \delta_{2} .
$$

To be specific, by negation, we have

$$
\text { meas }\left\{t \in \mathbb{R}: a(t)\left|q^{*}(t)\right|^{\gamma} \geq \frac{1}{n}\right\}=0
$$

for all $n \in \mathbb{N}$. Then by (7), one has

$$
0 \leq \int_{\mathbb{R}} a(t)\left|q^{*}\right|^{\gamma+2} d t \leq \frac{1}{n}\left\|q^{*}\right\|_{L^{2}}^{2} \leq \frac{\eta_{2}^{2}}{n}\left\|q^{*}\right\|^{2}=\frac{\eta_{2}^{2}}{n} \longrightarrow 0,
$$

as $n \rightarrow+\infty$, which contradicts that $\left\|q^{*}\right\|=1$. Therefore, (37) holds. Thus, define

$$
\begin{aligned}
\Omega_{0} & =\left\{t \in \mathbb{R}: a(t)\left|q^{*}(t)\right|^{\gamma} \geq \delta_{1}\right\}, \\
\Omega_{m} & =\left\{t \in \mathbb{R}: a(t)\left|q_{m}(t)\right|^{\gamma}<\frac{1}{m}\right\},
\end{aligned}
$$

and $\Omega_{m}^{c}=\mathbb{R} \backslash \Omega_{m}$. Combining (35) and (37), one has

$$
\begin{aligned}
\operatorname{meas}\left(\Omega_{m} \cap \Omega_{0}\right) & =\text { meas }\left(\Omega_{0} \backslash\left(\Omega_{m}^{c} \cap \Omega_{0}\right)\right) \\
& \geq \text { meas } \Omega_{0}-\text { meas }\left(\Omega_{m}^{c} \cap \Omega_{0}\right) \\
& \geq \delta_{2}-\frac{1}{m},
\end{aligned}
$$

for all $m \in \mathbb{N}$. Selecting $m>\max \left\{2 / \delta_{1}, 2 / \delta_{2}\right\}$, we have

$$
\delta_{1}-\frac{1}{m} \geq \frac{1}{2} \delta_{1}, \quad \delta_{2}-\frac{1}{m} \geq \frac{1}{2} \delta_{2} .
$$

Then, one gets

$$
\begin{aligned}
& a(t)\left|q_{m}(t)-q^{*}(t)\right|^{\gamma} \\
& \quad \geq a(t)\left|q^{*}(t)\right|^{\gamma}-a(t)\left|q_{m}(t)\right|^{\gamma} \\
& \quad \geq \delta_{1}-\frac{1}{m} \geq \frac{1}{2} \delta_{1}, \quad \forall t \in \Omega_{m} \cap \Omega_{0},
\end{aligned}
$$

which implies that

$$
\begin{aligned}
\int_{\mathbb{R}} a(t)\left|q_{m}-q^{*}\right|^{\gamma} d t & \geq \int_{\Omega_{m} \cap \Omega_{0}} a(t)\left|q_{m}-q^{*}\right|^{\gamma} d t \\
& \geq \frac{1}{2} \delta_{1} \cdot \operatorname{meas}\left(\Omega_{m} \cap \Omega_{0}\right) \\
& \geq \frac{1}{2} \delta_{1} \cdot\left(\delta_{2}-\frac{1}{m}\right) \geq \frac{1}{4} \delta_{1} \delta_{2},
\end{aligned}
$$

for $m>\max \left\{2 / \delta_{1}, 2 / \delta_{2}\right\}$, which is in contradiction to (36). Hence, (22) holds. Define

$$
\Omega_{q}=\left\{t \in \mathbb{R}: a(t)|q(t)|^{\gamma} \geq \varepsilon_{1}\|q\|^{\gamma}\right\}, \quad \forall q \in Y_{k} .
$$

It follows from $\left(W_{5}\right),(7),\left(W_{4}\right)$, and (22) that

$$
\begin{aligned}
I(q) & =\frac{1}{2}\|q\|^{2}-\int_{\mathbb{R}} W(t, q) d t \\
& \leq \frac{1}{2}\|q\|^{2}-\int_{\Omega_{q}} W(t, q) d t \\
& \leq \frac{1}{2}\|q\|^{2}-c_{0} \int_{\Omega_{q}} a(t)|q|^{\gamma} d t \\
& \leq \frac{1}{2}\|q\|^{2}-c_{0} \varepsilon_{1}\|q\|^{\gamma} \cdot \operatorname{meas}\left(\Omega_{q}\right) \\
& \leq \frac{1}{2}\|q\|^{2}-c_{0} \varepsilon_{1}^{2}\|q\|^{\gamma},
\end{aligned}
$$


for all $q \in Y_{k}$ with $\|q\| \leq \delta_{0} / \eta_{\infty}$. Since $1 \leq \gamma<2$, there exists $\rho_{k}>r_{k}>0$ such that

$$
b_{k}=\max _{q \in Y_{k},\|q\|=r_{k}} I(q)<0
$$

Step 3. Condition $\left(B_{5}\right)$ holds. Since it is similar to the second step in the proof of Theorem 2, we omit it here.

At last, it is standard to verify that condition $\left(B_{1}\right)$ holds and $I$ is invariant. So, Theorem 4 is proved by Lemma 13 immediately.

Proof of Theorem 6. Since the proof of Theorem 6 is exactly similar to the proof of Theorem 4, we omit it here.

\section{Conflict of Interests}

The author declares that there is no conflict of interests regarding this work.

\section{Acknowledgments}

The paper is supported by General Project of Educational Department in Sichuan (no. 13ZB0182) and Doctor Research Foundation of Southwest University of Science and Technology (no. 11zx7130).

\section{References}

[1] M. Izydorek and J. Janczewska, "Homoclinic solutions for a class of the second order Hamiltonian systems," Journal of Differential Equations, vol. 219, no. 2, pp. 375-389, 2005.

[2] C. O. Alves, P. C. Carrião, and O. H. Miyagaki, "Existence of homoclinic orbits for asymptotically periodic systems involving Duffing-like equation," Applied Mathematics Letters, vol. 16, no. 5, pp. 639-642, 2003.

[3] P. C. Carrião and O. H. Miyagaki, "Existence of homoclinic solutions for a class of time-dependent Hamiltonian systems," Journal of Mathematical Analysis and Applications, vol. 230, no. 1, pp. 157-172, 1999.

[4] P. H. Rabinowitz, "Homoclinic orbits for a class of Hamiltonian systems," Proceedings of the Royal Society of Edinburgh A, vol. 114, no. 1-2, pp. 33-38, 1990.

[5] Y. H. Ding, "Existence and multiplicity results for homoclinic solutions to a class of Hamiltonian systems," Nonlinear Analysis: Theory, Methods \& Applications, vol. 25, no. 11, pp. 1095-1113, 1995.

[6] W. Omana and M. Willem, "Homoclinic orbits for a class of Hamiltonian systems," Differential and Integral Equations, vol. 5, no. 5, pp. 1115-1120, 1992.

[7] Z.-Q. Ou and C.-L. Tang, "Existence of homoclinic solution for the second order Hamiltonian systems," Journal of Mathematical Analysis and Applications, vol. 291, no. 1, pp. 203-213, 2004.

[8] W. M. Zou and S. J. Li, "Infinitely many homoclinic orbits for the second-order Hamiltonian systems," Applied Mathematics Letters, vol. 16, no. 8, pp. 1283-1287, 2003.

[9] J. Ding, J. Xu, and F. Zhang, "Even homoclinic orbits for super quadratic Hamiltonian systems," Mathematical Methods in the Applied Sciences, vol. 33, no. 14, pp. 1755-1761, 2010.
[10] P. Korman and A. C. Lazer, "Homoclinic orbits for a class of symmetric Hamiltonian systems," Electronic Journal of Differential Equations, vol. 1994, no. 1, pp. 1-10, 1994.

[11] Y. Lv and C.-L. Tang, "Existence of even homoclinic orbits for second-order Hamiltonian systems," Nonlinear Analysis: Theory, Methods \& Applications, vol. 67, no. 7, pp. 2189-2198, 2007.

[12] Z. Zhang and R. Yuan, "Homoclinic solutions for a class of nonautonomous subquadratic second-order Hamiltonian systems," Nonlinear Analysis: Theory, Methods \& Applications, vol. 71, no. 9, pp. 4125-4130, 2009.

[13] J. Sun, H. Chen, and J. J. Nieto, "Homoclinic solutions for a class of subquadratic second-order Hamiltonian systems," Journal of Mathematical Analysis and Applications, vol. 373, no. 1, pp. 20 29, 2011.

[14] L. Yang, H. Chen, and J. Sun, "Infinitely many homoclinic solutions for some second order Hamiltonian systems," Nonlinear Analysis: Theory, Methods \& Applications, vol. 74, no. 17, pp. 6459-6468, 2011.

[15] X. Lv and J. Jiang, "Existence of homoclinic solutions for a class of second-order Hamiltonian systems with general potentials," Nonlinear Analysis: Real World Applications, vol. 13, no. 3, pp. 1152-1158, 2012.

[16] S. Lu, "Homoclinic solutions for a nonlinear second order differential system with $p$-Laplacian operator," Nonlinear Analysis: Real World Applications, vol. 12, no. 1, pp. 525-534, 2011.

[17] W. Zou, "Variant fountain theorems and their applications," Manuscripta Mathematica, vol. 104, no. 3, pp. 343-358, 2001.

[18] P. H. Rabinowitz, Minimax Methods in Critical Point Theory with Applications to Differential Equations, vol. 65 of CBMS Regional Conference Series in Mathematics, American Mathematical Society, Providence, RI, USA, 1986.

[19] M. Willem, Minimax Theorems, vol. 24 of Progress in Nonlinear Differential Equations and Their Applications, Birkhäuser, Boston, Mass, USA, 1996. 


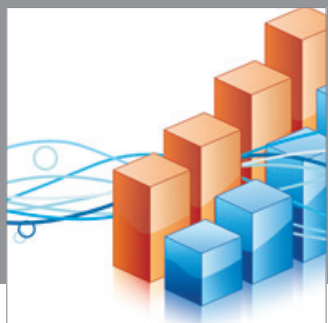

Advances in

Operations Research

mansans

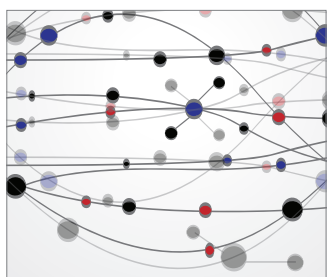

The Scientific World Journal
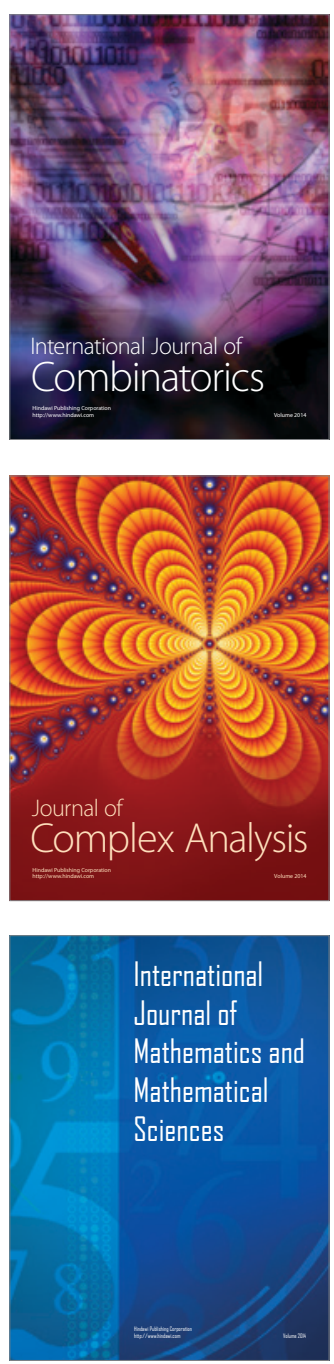
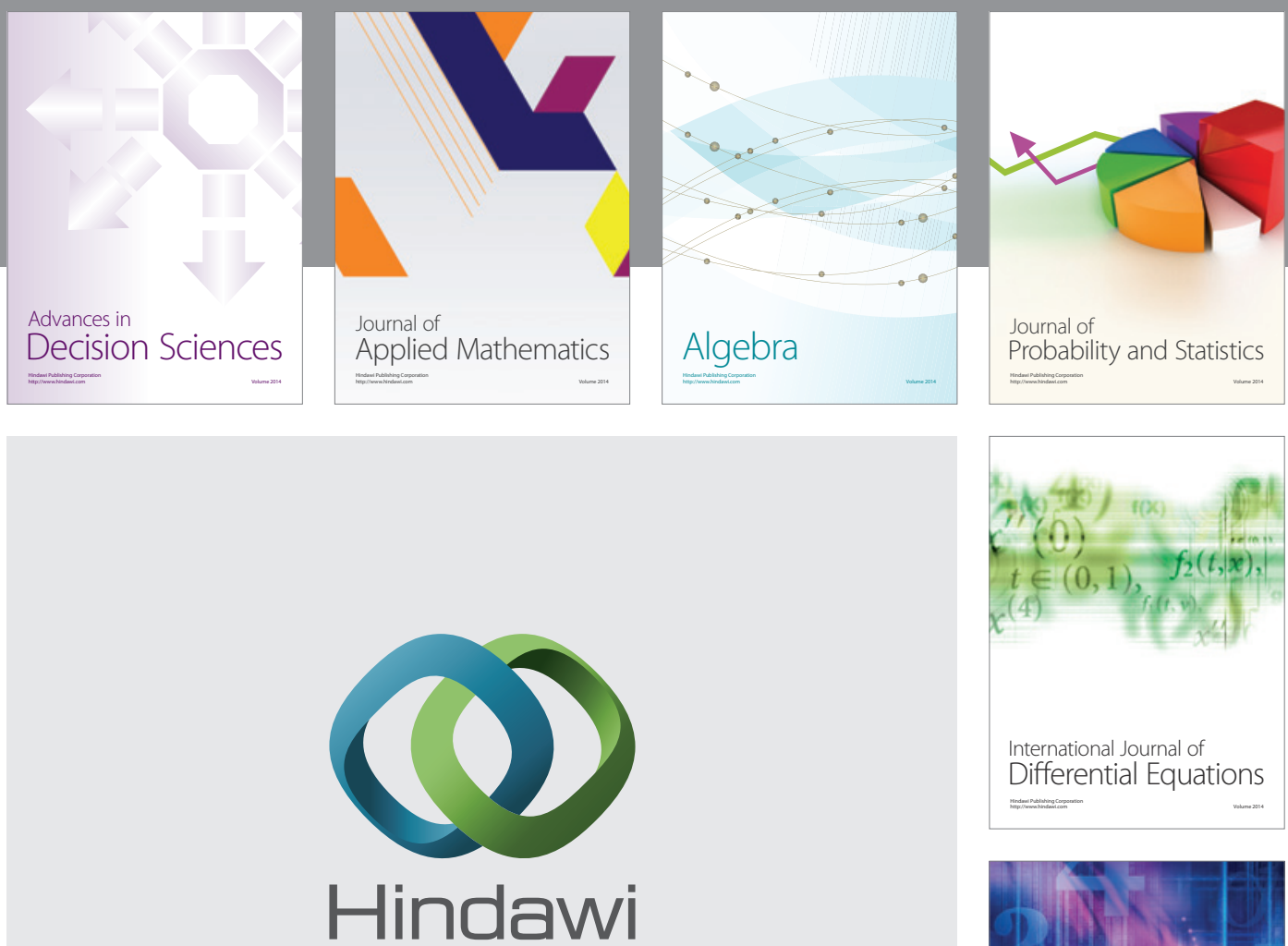

Submit your manuscripts at http://www.hindawi.com
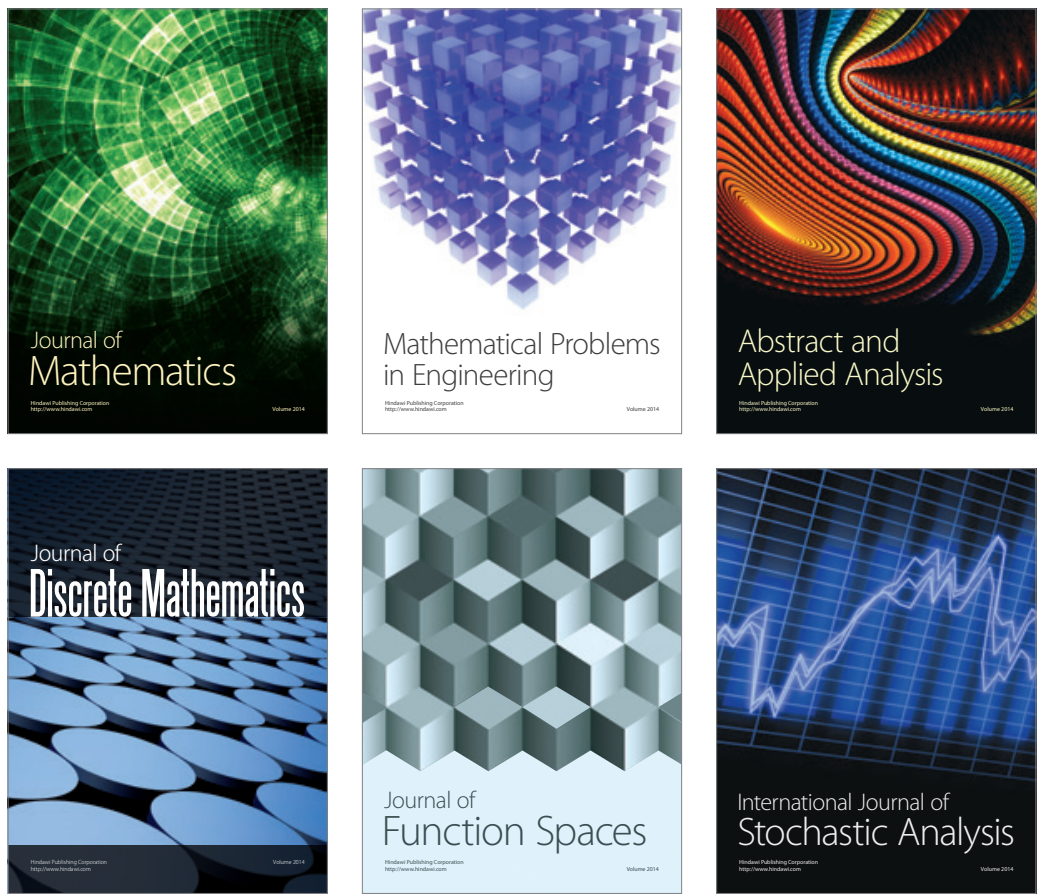

Journal of

Function Spaces

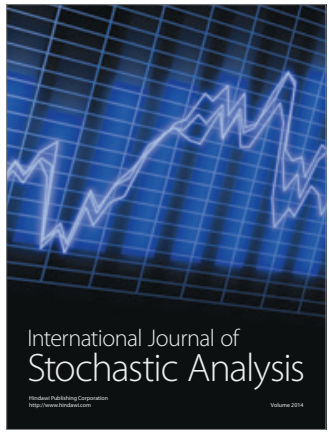

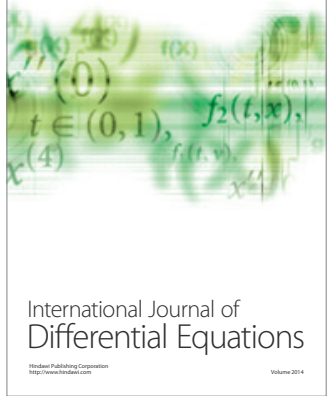
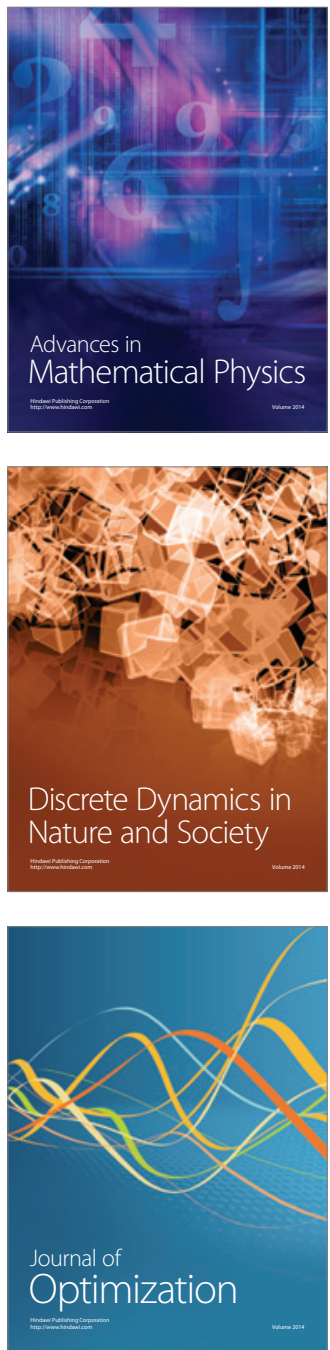